\title{
Dynamically Weighted Clique Evolution Model in Clique Networks
}

\author{
Zhang-Wei Li, ${ }^{1}$ Xu-Hua Yang, ${ }^{1}$ Feng-Ling Jiang, ${ }^{1}$ Guang Chen, ${ }^{1}$ \\ Guo-Qing Weng, ${ }^{1}$ and Mei $\mathrm{Zhu}^{2}$ \\ ${ }^{1}$ College of Computer Science and Technology, Zhejiang University of Technology, Hangzhou 310023, China \\ ${ }^{2}$ College of Information Engineering, Zhejiang University of Science \& Technology, Hangzhou 310023, China
}

Correspondence should be addressed to Xu-Hua Yang; xhyang@zjut.edu.cn

Received 2 February 2013; Accepted 26 February 2013

Academic Editor: Shengyong Chen

Copyright (C) 2013 Zhang-Wei Li et al. This is an open access article distributed under the Creative Commons Attribution License, which permits unrestricted use, distribution, and reproduction in any medium, provided the original work is properly cited.

\begin{abstract}
This paper proposes a weighted clique evolution model based on clique (maximal complete subgraph) growth and edge-weight driven for complex networks. The model simulates the scheme of real-world networks that the evolution of networks is likely to be driven by the flow, such as traffic or information flow needs, as well as considers that real-world networks commonly consist of communities. At each time step of a network's evolution progress, an edge is randomly selected according to a preferential scheme. Then a new clique which contains the edge is added into the network while the weight of the edge is adjusted to simulate the flow change brought by the new clique addition. We give the theoretical analysis based on the mean field theory, as well as some numerical simulation for this model. The result shows that the model can generate networks with scale-free distributions, such as edge weight distribution and node strength distribution, which can be found in many real-world networks. It indicates that the evolution rule of the model may attribute to the formation of real-world networks.
\end{abstract}

\section{Introduction}

With the rapid development of information and other sciences, today connections between people have been widely extended and communications within systems which can be abstracted into networks can be found all round us. Nowadays, human's living and producing activities depend much wider and deeper on those complex network systems than ever before [1-3]. How to ensure those systems to work in a secure and efficient way is a big deal $[4,5]$. Beginning from the studies on a small-world [6], scale-free [7], and navigable [8] phenomenon in common networks, a significant concern and attention have been attracted from different fields to establish and study a new interdisciplinary subject, the network science.

Just like the three phenomena mentioned above, realworld networks have been found that they commonly evolve by simple mechanisms and gradually form [7] into the complex systems observed by us. Revealing those simple rules is one important aspect of network science research, as those rules can help us better understand and improve the properties and behaviors of networks. In many of those researches, simple unweighted network models were widely used for analysis $[7,9,10]$. However, sometimes it is not sufficient, as simply using an edge to represent the connections between nodes in network will lose some features we need to be concerned, such as the importance of nodes, the traffic flow on edges. As a result, many weighted evolution network models [11-15] were proposed. Among them, two famous and widely accepted examples are the BBV model [12] and the DM model [13]. The two models have successfully reproduced the scalefree phenomena while they proposed that a network's evolution should be driven by traffic flow needs, which is similar to the situation in many real-world networks, like road network [16], and mobile agent network [17].

Researches have also revealed that communities (communities are known as kinds of subgraphs of networks that the connections within themselves are tight, while connections between them and other parts of networks are sparse) exist widely in complex networks [18-21]. For instance, social 
networks $[3,22]$ include communities such as families and clubs and bus transport networks [23] consist of communities representing bus routes. Usually, these networks evolve with units of communities and communities may overlap with each other. For example, the bus transport network expands by adding communities which are comprised of stations in one bus route. Obviously, there exist overlapping communities in the network because an arbitrary station may be included in several bus routes [24-26].

On the basis, in this paper we propose a new weighted network evolution model based on edge weight driven (where edge weight can be viewed as to represent the flow or other meaningful things of a connection) and clique [19] growth (where cliques are maximal complete sub graphs in a network, used in this paper to mimic communities in realworld networks). Through theoretical analysis and numerical simulation of the model, it is proven that our model can reproduce scale-free distributions [7] while it involves addition of cliques. The remainder of the paper is organized as follows. In section two, we will present our model. In sections three and four, some theoretical analysis and numerical simulation about the model will be introduced. Finally in section five, we will give our discussion and conclusion.

\section{Presentation of the Evolution Model of Clique Growth and Edge-Weight Driven}

We present our new model in this section. At the beginning time step $t=0$, the model starts with a clique (a maximal complete subgraph) denoted by $c_{0}$, which consists of $m_{0}$ vertices. The weight of each edge in the clique is initially set as $w_{0}$. Then the network will be expanded continuously as well as change its inner edges' weight on the meanwhile dynamically based on the scheme of clique growth and edge-weight driven. The specific generating progress for each time step of the evolution is as follows.

(1) Preferential selection: randomly select from the network an edge according to a preferential scheme based on edge weight. Specifically, the probability of an edge being selected is proportional to its edge weight. That is, if $w_{i j}$ denotes the weight of an edge connecting nodes $i$ and $j, P_{i j}$ denotes the probability for the edge being selected, then $P_{i j}=w_{i j} / \sum_{m, n} w_{m n}$.

(2) Clique growth: add a new clique consisting of $m_{2}+2$ nodes into the network, while rule that the new clique must contain the edge selected by step (1). For the edges except the selected edge, the weight is set as $w_{0}$.

(3) Edge weight adjustment: give the selected edge a weight increment of $\Delta$, which makes the weight of this edge change from $w_{i j}$ to $w_{i j}+\Delta$.

It is worth noting that the increment of $\Delta$ is introduced in the above step (3), as in real situations; when a community is added into a network, it will bring some influence on the original network. For example, in bus transport networks, when a bus route is added, the passenger flow of the original network will be changed.

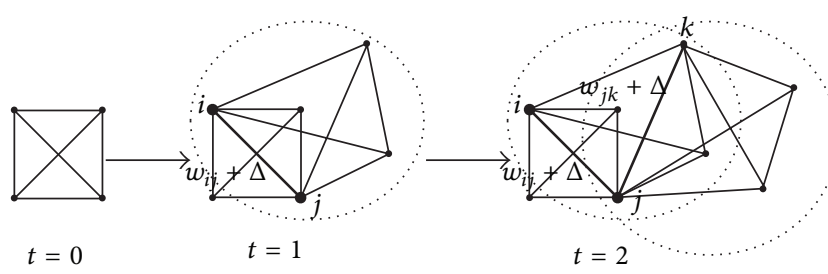

FIGURE 1: Illustration example of the evolution process.

We give an illustrative example for the evolution process in Figure 1. The parameters are set as $m_{0}=4, m_{2}=2$, and $w_{0}=1$. At the time step $t=0$, the initial network is constructed as a clique which is composed of $m_{0}=4$ vertices. At the time step $t=1$, a new clique is added into the network (dotted lines). The edge connecting nodes $i$ and $j$ is selected from the network based on the edge weight preferential scheme. Two new nodes are added into the network to constitute the clique. After the addition, we set the weights of edges expecting the selected edge $w_{0}$ and change the weight of the selected edge into $w_{i j}+\Delta$. At the time step $t=2$, another clique is added into the network and the rule the same.

\section{Theoretical Analysis of the Model with a Brief Simulation}

We give some theoretical analysis of the model based on mean field theory [27] in this section. The analysisis concerned with the edge weight distribution and the node strength distribution, which are two important indices to reflect the structure properties in weighed networks. The edge weight is widely used to depict the specific differences of connections in networks, such as the intimacy between friends, the distance between places, and the traffic flow capacity of a road, and so forth. On the other side, the node strength is used to depict the importance of the nodes which may represent people, routers, and so forth, in networks. Usually, the edge weight and the node strength have kinds of relationship. In many previous researches, the relationship is defined as $s_{i}=$ $\sum_{j} w_{i j}[11,12]$ which is similar to the real-world situation, where $s_{i}$ denotes the strength of a specific node $i$. In this paper, we adapt this relationship for further analysis.

As can be seen, when the incensement parameter is set as $\Delta=0$, the new model will behave similarly like the BA scalefree model [7]. The only difference is that new cliques instead of new nodes are added into networks during their evolution. So in this situation, the theoretical analysis of our model should resemble BAs. But when nonzero $\Delta$ is introduced, we need to make some changes in order to obtain correct results.

In each time step, the evolution process of our model begins with a preferential selection of an edge, followed by an addition of a new clique. As a result, when an edge connecting nodes $i$ and $j$ is selected, the weight $w_{i j}$ will obey (1) according to the mean-field theory:

$$
\frac{d w_{i j}}{d t}=\frac{w_{i j}}{\sum_{m, n} w_{m n}} \Delta .
$$


Given $t_{i j}$ denotes the time step when this edge was added into the network, then the initial condition

$$
w_{i j}\left(t_{i j}\right)=w_{0}
$$

for (1) should be satisfied. According to the model, the total weight being added into the network in each time step is equal to

$$
\Gamma=\left[\frac{\left(m_{2}+2\right)\left(m_{2}-1\right)}{2}-1\right] w_{0}+\Delta
$$

So

$$
\sum_{m, n} w_{m n} \approx \Gamma t
$$

when $t \rightarrow \infty$.

Solving (1), (2), and (3), we can obtain the specific formula of $w_{i j}(t)$. For example, with the scenario in Figure 1 , the parameters are $m_{0}=4, m_{2}=2$, and $w_{0}=1$, so we get $\Gamma=5+\Delta$, and the formation of $w_{i j}(t)$ can be obtained as

$$
w_{i j}(t)=\left(\frac{t}{t_{i j}}\right)^{\Delta /(5+\Delta)} .
$$

With solved $w_{i j}(t)$, the edge weight distribution can be further derived. Here, also taking Figure 1 scenario as an example, we can get

$$
t_{i j}=t w_{i j}^{-(5+\Delta) / \Delta}
$$

According to the mean field theory, the equations can be transformed into

$$
\begin{aligned}
P\left(w_{i j}<w\right) & =P\left(t_{i j}>t w^{-(5+\Delta) / \Delta}\right) \\
& =1-P\left(t_{i j} \leq t w^{-(5+\Delta) / \Delta}\right) \\
& =1-w^{-(5+\Delta) / \Delta},
\end{aligned}
$$

where $P\left(w_{i j}<w\right)$ is the proportion of edges in a network whose weight is less than $w$ and $P\left(t_{i j}>t\right)\left(P\left(t_{i j} \leq t\right)\right)$ is the proportion of edges which were added into the network after, before, or at the time step $t$.

Further, we can get the result as

$$
\begin{gathered}
p(w, t)=\frac{\partial\left(P\left(w_{i j}(t)<w\right)\right)}{\partial w}=\frac{(5+\Delta)}{\Delta \times w^{-(2 \Delta+5) / \Delta},} \\
p(w)=\lim _{t \rightarrow \infty} p(w, t)=\frac{1}{\beta} w^{-\Upsilon} \sim w^{-\gamma},
\end{gathered}
$$

where $p(w)$ is the edge weight distribution, $\beta=\Delta /(5+\Delta)$, and $\gamma=(2 \Delta+5) / \Delta$.

Equation (9) shows that, the edge weight distribution of our model is a kind of scale-free distribution. We conduct a numerical simulation for the same parameters in the scenario of Figure 1 to verify the theoretical result. The value of $\Delta$ is set as $\Delta=3$. The network evolution progress stops at $t=10^{4}$. We

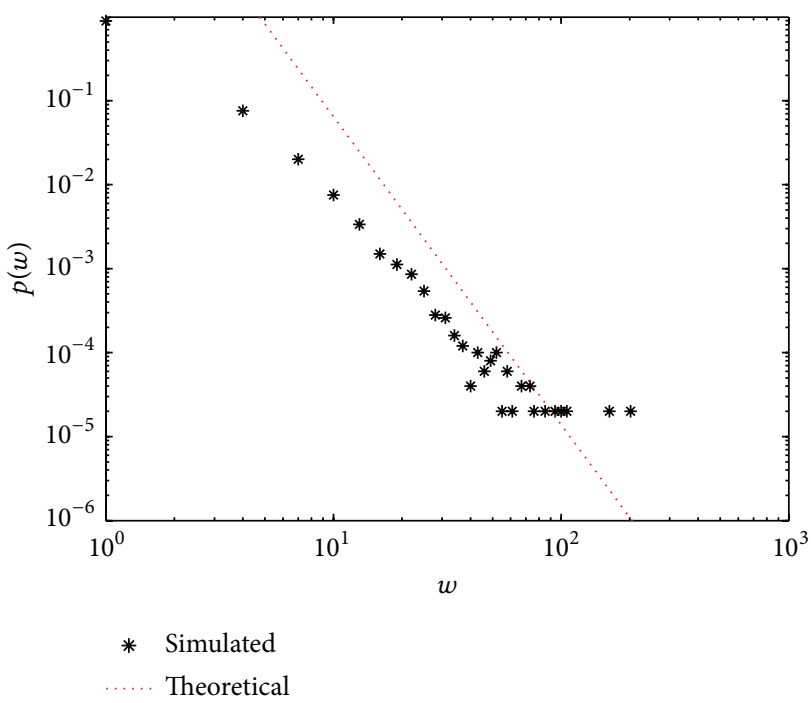

FIGURE 2: The edge weight distribution of the simulated network.

show the resulting edge weight distribution for the generated network in Figure 2. From the theoretical analysis, we know that exponent $\gamma$ of the scale-free distribution should be equal to $(2 \Delta+5) / \Delta \approx 3.67$. Figure 2 shows that simulation result agrees with the theoretical analysis. Although there is some deviation at the head of the distribution, the error should be decreased with the increase of evolution time.

Similarly, for node $i$, when an edge connecting this node with other node $j$ is selected in one time step, its strength will increase by $m_{2}$ (brought by the new added clique) $+\Delta$ (brought by the weight adjustment). Summing up all the possible increments brought when one of the edges emanated from node $i, d s_{i} / d t$ can be derived as

$$
\begin{aligned}
\frac{d s_{i}}{d t} & =\sum_{j} \frac{w_{i j}}{\sum_{m, n} w_{m n}}\left(m_{2}+\Delta\right) \\
& =\sum_{j} \frac{w_{i j}}{\Gamma t}\left(m_{2}+\Delta\right) \\
& =\frac{s_{i}}{\Gamma t}\left(m_{2}+\Delta\right) .
\end{aligned}
$$

Here we have the initial condition $s_{i}\left(t_{i}\right)=1$, where $t_{i}$ denotes the time when node $i$ is added into network. After solving (10) with the initial condition, we can obtain the specific formula of $s_{i}(t)$. For Figure 1 scenario, the result should be

$$
s_{i}=\left(\frac{t}{t_{i}}\right)^{5 /(4+2 \Delta)} .
$$

Similarly with the derivation of the edge weight distribution, we can obtain the node strength distribution denoted as $p(s)$ with the help of (11). The result for Figure 1 scenario is

$$
p(s) \sim s^{-\gamma},
$$

where $\gamma=(3 \Delta+4) / 5$. 


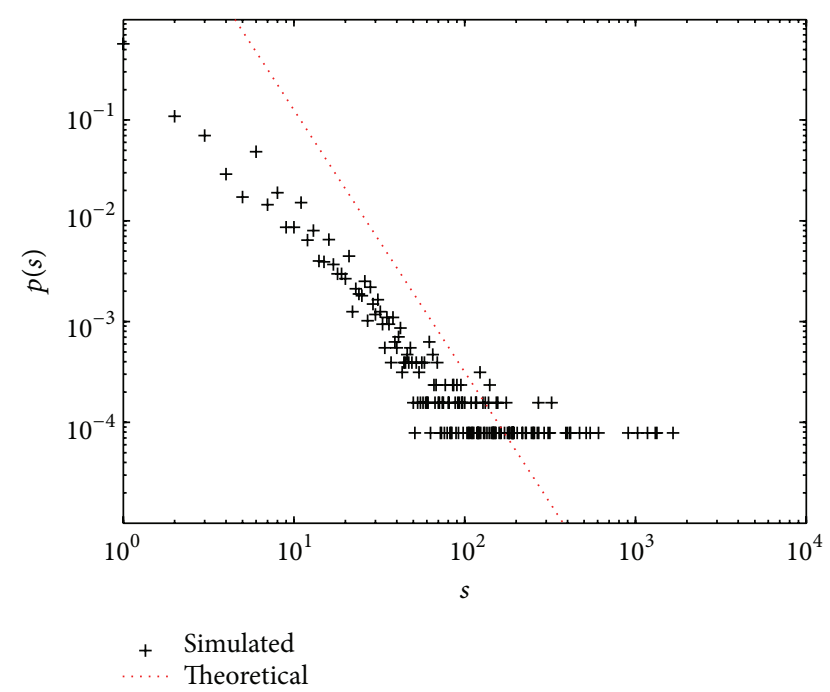

FIGURE 3: The node strength distribution of the simulated network.

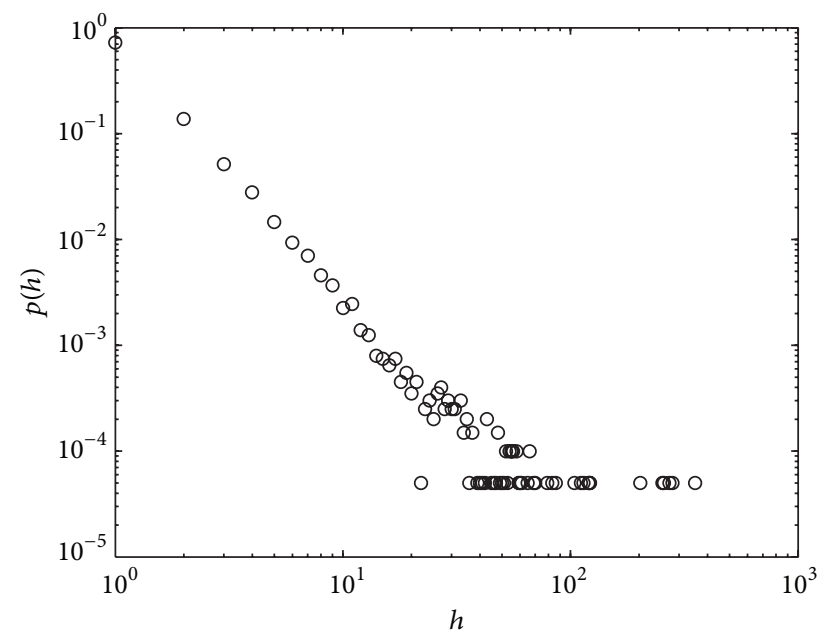

FIGURE 4: The distribution of the number of cliques that a node belongs to.

Equation (12) indicates that the node strength distribution is also a kind of scale-free distributions. The node strength distribution for the simulated network is presented in Figure 3. The theoretical value for the scale-free exponent $\gamma$ equals 2.6. From Figure 3, we can see that the simulated node strength distribution agrees well with the theoretical result.

\section{More Detailed Information about the Simulation}

In this section, we give more information about the simulation mentioned in the above section. The result is presented from Figure 4 to Figure 8. Figures 4 and 5 indicate that the distributions of the number of cliques that a node belongs to and the node degree distribution are kind of scalefree distributions. The reason for this phenomenon is that node strength should have some linear relationship with the

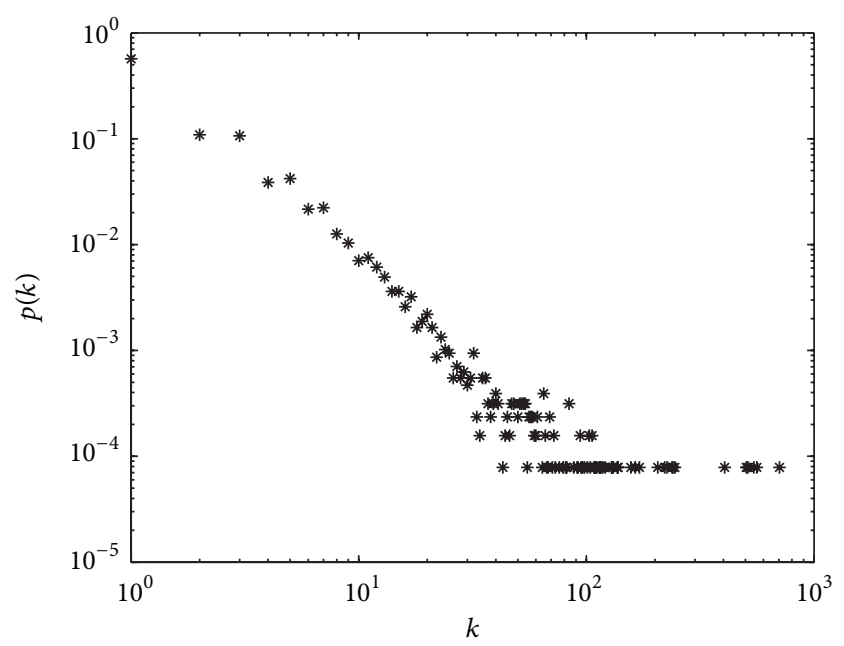

FIgURE 5: The node degree distribution.

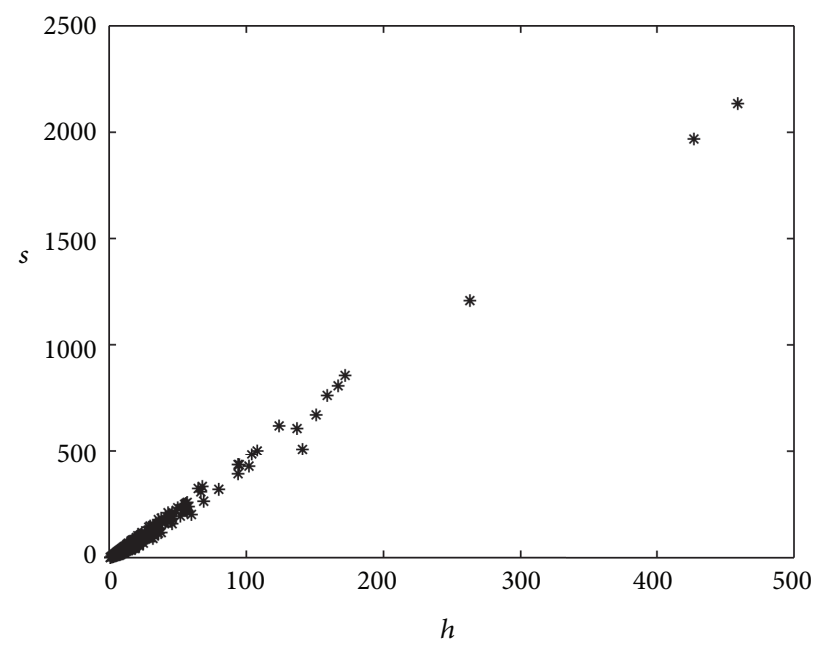

FIGURE 6: The correlation between node strength $(s)$ and number of cliques nodes belong to $(h)$.

node degree as well as with the number of cliques that a node belongs to. These relationships have been verified with Figures 6 and 7. Figures 8 and 9 indicate that the weight of an edge and the strength of a node in the network increase logarithmically with time. This is in accordance with the theoretical result showed in (5) and (11).

\section{Conclusion}

In this paper, we have proposed a new weighted evolution model for complex networks based on clique growth and edge weight-driven. The result has proven that our model can generate networks consisting of cliques and also with scalefree distributions, such as edge weight distribution, and node strength distribution.

Using weighted and community network models can help us figure out the actions in real-world network much better. The work of this paper is just a preliminary exploration of 


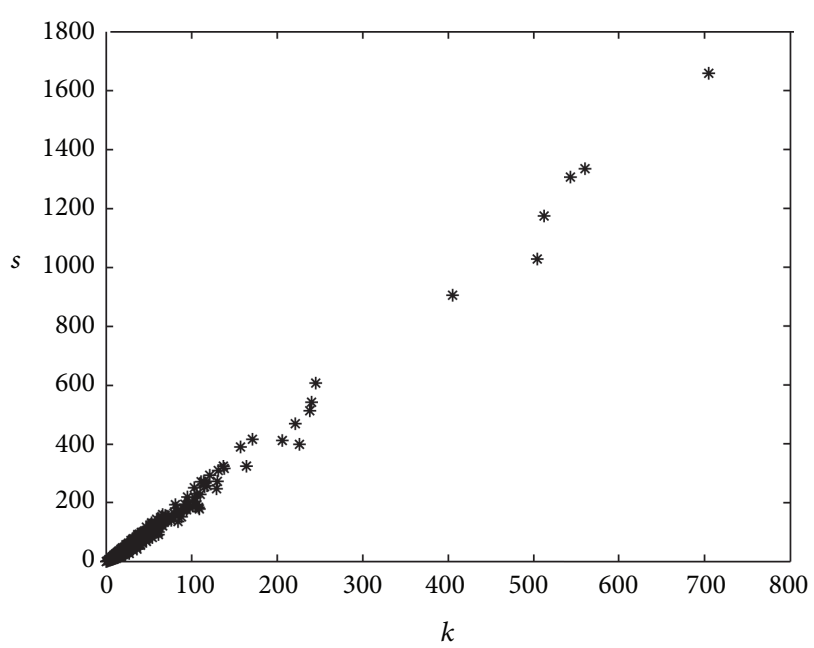

FIGURE 7: The correlation between node strength $(s)$ and node degree $(k)$.

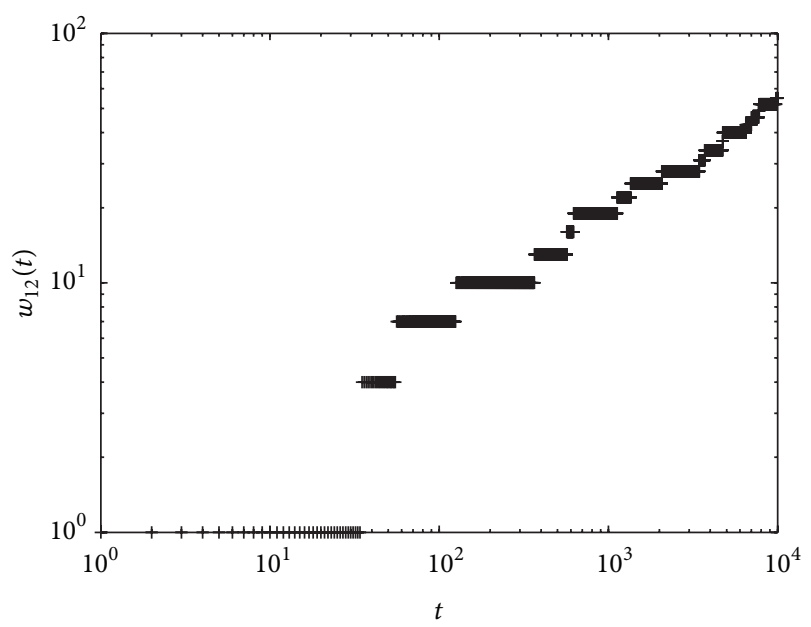

Figure 8: The change of the weight of the very first edge $\left(w_{12}\right)$ with time $(t)$.

the evolution mechanisms of real-world networks. The realworld networks are much more complicated and diverse although the rules behind them may be simple, but the rules are coupled with each other. For instance, the real-world networks sometimes may grow by either single nodes or communities at a specific time step, while sometimes they may reduce nodes or communities during their evolution as well as take some inner structure adjustment. In this paper, we have only considered some static properties of networks. The fact is that the dynamical properties of networks are as equally important as the static ones. We hope to solve these problems in a good way. this will be our future research content.

\section{Acknowledgments}

The work is supported by the National Natural Science Foundation of China under Grant no. 60874080, the Commonweal Application Technique Research Project of Zhejiang Province under Grant no. 2012C23126, and the Natural

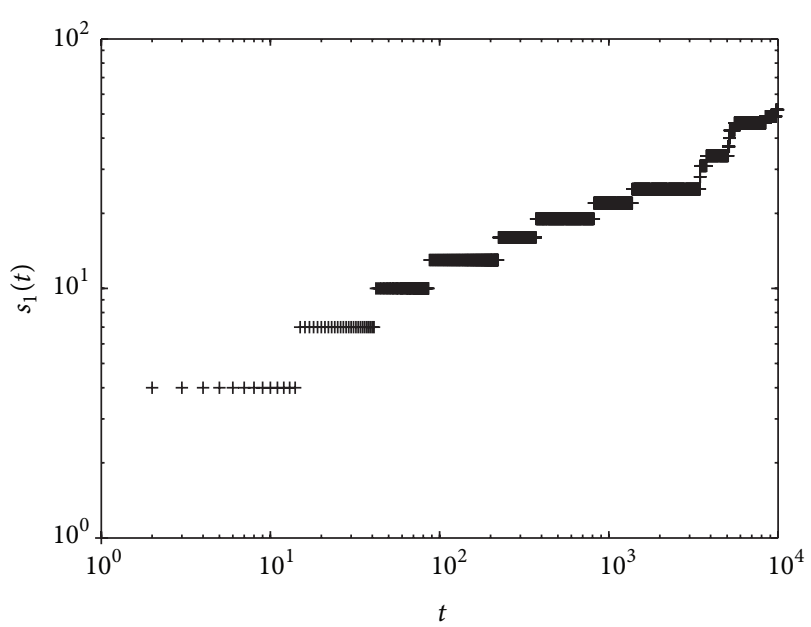

FIGURE 9: The change of the strength of the very first node $\left(s_{1}\right)$ with time $(t)$.

Science Foundation of Zhejiang Province of China under Grant no. LY12E07005.

\section{References}

[1] F. Schweitzer, G. Fagiolo, D. Sornette, F. Vega-Redondo, A. Vespignani, and D. R. White, "Economic networks: the new challenges," American Association for the Advancement of Science. Science, vol. 325, no. 5939, pp. 422-425, 2009.

[2] E. Bullmore and O. Sporns, "Complex brain networks: graph theoretical analysis of structural and functional systems," Nature Reviews Neuroscience, vol. 10, no. 3, pp. 186-198, 2009.

[3] A. L. Traud, P. J. Mucha, and M. A. Porter, "Social structure of Facebook networks," Physica A, vol. 391, no. 16, pp. 4165-4180, 2012.

[4] X.-H. Yang, G. Chen, B. Sun, S.-Y. Chen, and W.-L. Wang, "Bus transport network model with ideal $n$-depth clique network topology," Physica A, vol. 390, no. 23-24, pp. 4660-4672, 2011.

[5] J. Xue, M. Li, W. Zhao, and S. Y. Chen, "Bound maxima as a traffic feature under DDOS flood attacks," Mathematical Problems in Engineering, vol. 2012, Article ID 465613, 9 pages, 2012.

[6] D. J. Watts and S. H. Strogatz, "Collective dynamics of 'smallworld' networks," Nature, vol. 393, no. 6684, pp. 440-442, 1998.

[7] A.-L. Barabási and R. Albert, "Emergence of scaling in random networks," American Association for the Advancement of Science, vol. 286, no. 5439, pp. 509-512, 1999.

[8] J. M. Kleinberg, "Navigation in a small world," Nature, vol. 406, no. 6798 , p. $845,2000$.

[9] Z.-J. Bao and Y.-J. Cao, "Cascading failures in local-world evolving networks," Journal of Zhejiang University A, vol. 9, no. 10, pp. 1336-1340, 2008.

[10] Y.-X. Li, X.-G. Jin, F.-S. Kong, and H.-L. Luo, "Strategic games on a hierarchical network model," Journal of Zhejiang University A, vol. 9, no. 2, pp. 271-278, 2008.

[11] A. Barrat, M. Barthélemy, R. Pastor-Satorras, and A. Vespignani, "The architecture of complex weighted networks," Proceedings of the National Academy of Sciences of the United States of America, vol. 101, no. 11, pp. 3747-3752, 2004. 
[12] A. Barrat, M. Barthélemy, and A. Vespignani, "Weighted evolving networks: coupling topology and weight dynamics," Physical Review Letters, vol. 92, no. 22, Article ID 228701, 4 pages, 2004.

[13] S. N. Dorogovtsev, J. F. F. Mendes, and A. N. Samukhin, "Sizedependent degree distribution of a scale-free growing network," Physical Review E, vol. 63, no. 6, part 1, Article ID 062101, 2001.

[14] G. H. Wen, Z. S. Duan, G. R. Chen, and X. M. Geng, "A weighted local-world evolving network model with aging nodes," Physica A, vol. 390, no. 21-22, pp. 4012-4026, 2011.

[15] H. H. Jo, R. K. Pan, and K. Kaski, "Emergence of bursts and communities in evolving weighted networks," PLOS ONE, vol. 6, no. 8, Article ID e22687, 2011.

[16] F. Bono, E. Gutirrez, and K. Poljansek, "Road traffic: a case study of flow and path-dependency in weighted directed networks," Physica A, vol. 389, no. 22, pp. 5287-5297, 2010.

[17] H. X. Yang, W.-X. Wang, Y.-C. Lai, and B. H. Wang, "Trafficdriven epidemic spreading on networks of mobile agents," $E P L$, vol. 98, no. 6, Article ID 68003, 2012.

[18] M. E. J. Newman, "Fast algorithm for detecting community structure in networks," Physical Review E, vol. 69, no. 6, Article ID 066113, 2004.

[19] G. Palla, I. Derényi, I. Farkas, and T. Vicsek, "Uncovering the overlapping community structure of complex networks in nature and society," Nature, vol. 435, no. 7043, pp. 814-818, 2005.

[20] M. Rosvall and C. T. Bergstrom, "Maps of random walks on complex networks reveal community structure," Proceedings of the National Academy of Sciences of the United States of America, vol. 105, no. 4, pp. 1118-1123, 2008.

[21] C. Seshadhri, T. G. Kolda, and A. Pinar, "Community structure and scale-free collections of Erdos-Renyi graphs," Physical Review E, vol. 85, no. 5, Article ID 056109, 2012.

[22] T. Zhou, M. Medo, G. Cimini, Z. K. Zhang, and Y. C. Zhang, "Emergence of Scale-Free leadership structure in social recommender systems," PLoS ONE, vol. 6, no. 7, Article ID e20648, 2011.

[23] X.-H. Yang, G. Chen, B. Sun, S.-Y. Chen, and W.-L. Wang, "Bus transport network model with ideal $n$-depth clique network topology," Physica A, vol. 390, no. 23-24, pp. 4660-4672, 2011.

[24] S. Chen, W. Huang, C. Cattani, and G. Altieri, "Traffic dynamics on complex networks: a survey," Mathematical Problems in Engineering, vol. 2012, Article ID 732698, 23 pages, 2012.

[25] M. Li, W. Zhao, and S. Chen, "mBm-based scalings of traffic propagated in internet," Mathematical Problems in Engineering, vol. 2011, Article ID 389803, 21 pages, 2011.

[26] W. Huang and S.-Y. Chen, "Epidemic metapopulation model with traffic routing in scale-free networks," Journal of Statistical Mechanics, Article ID P12004, 19 pages, 2011.

[27] A. L. Barabási, R. Albert, and H. Jeong, "Mean-field theory for scale-free random networks," Physica A, vol. 272, no. 1-2, pp. 173-187, 1999. 


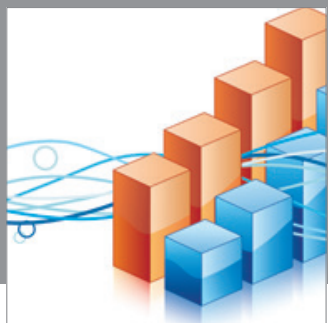

Advances in

Operations Research

mansans

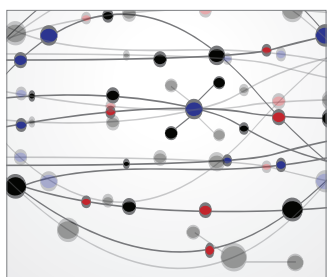

The Scientific World Journal
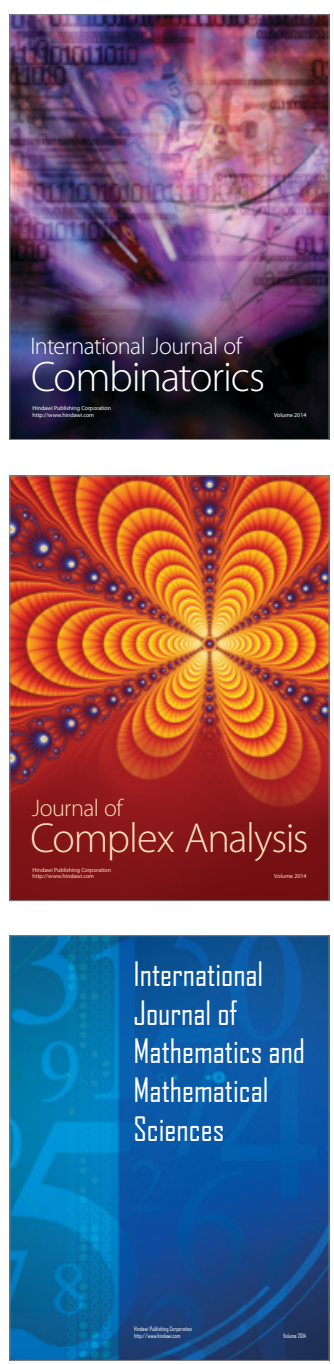
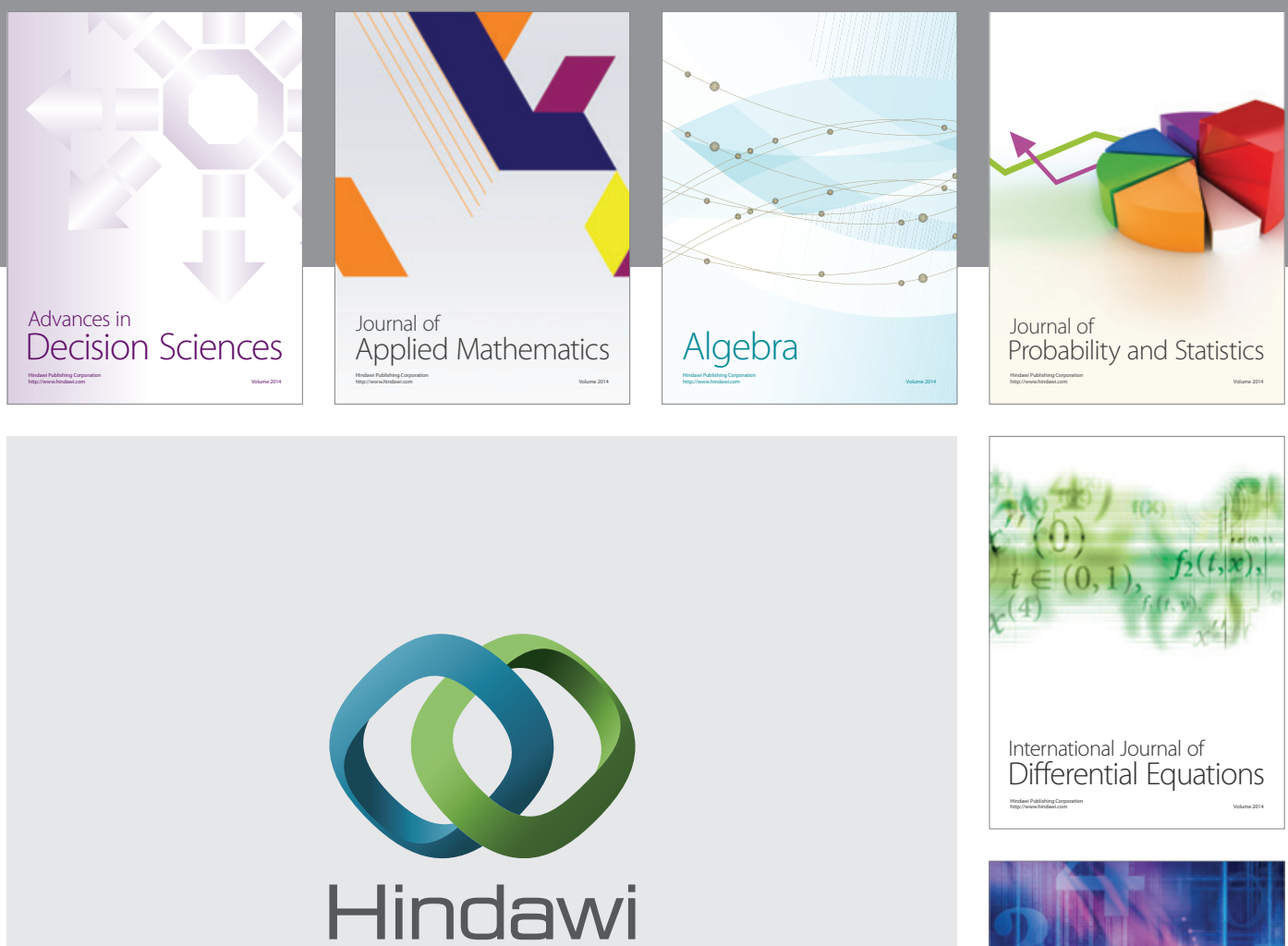

Submit your manuscripts at http://www.hindawi.com
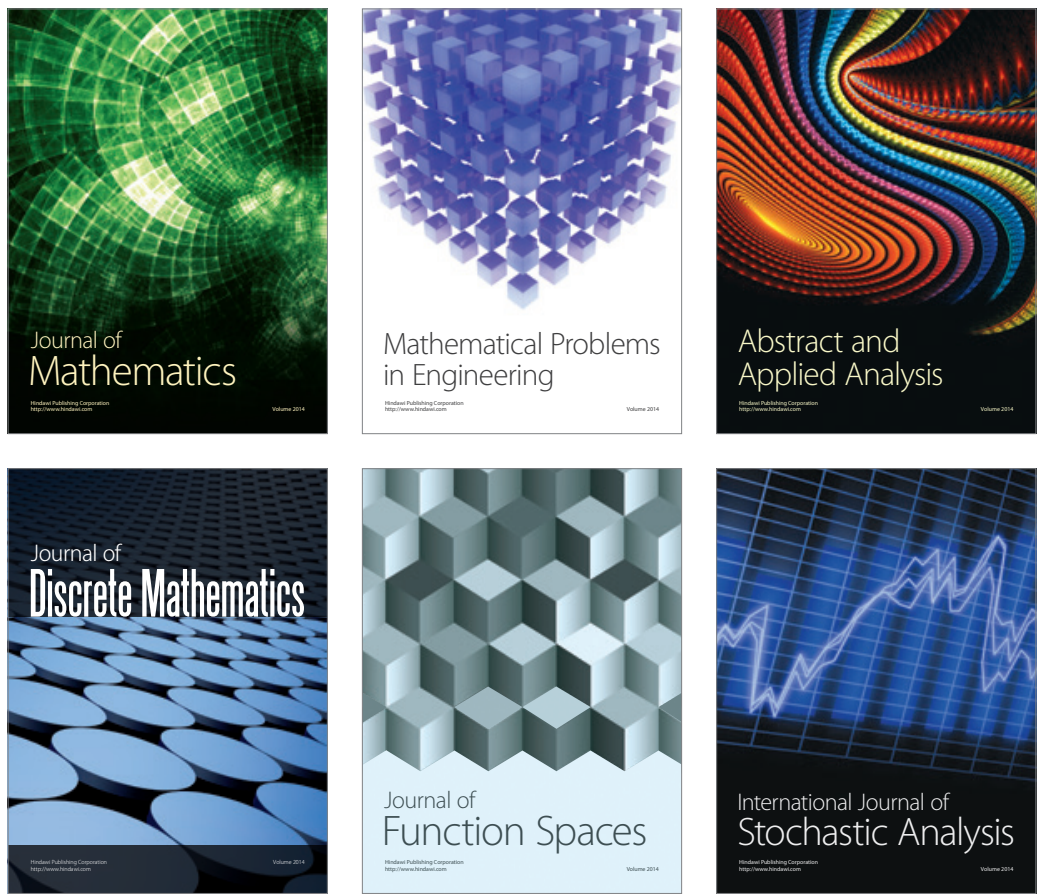

Journal of

Function Spaces

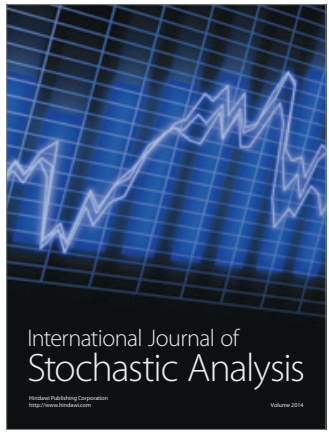

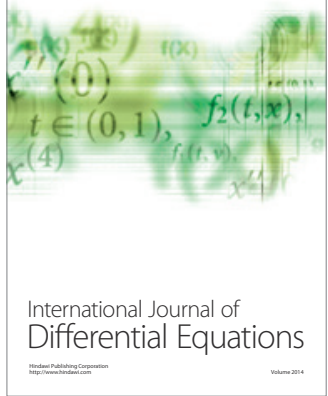
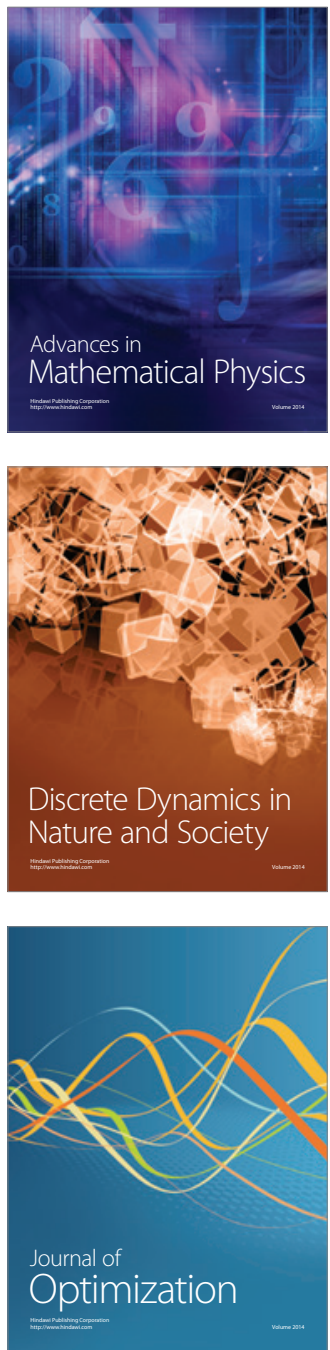